\title{
Temperature dependence of DNA translocations through solid-state nanopores
}

\author{
Daniel V Verschueren, Magnus P Jonsson and Cees Dekker
}

\section{Linköping University Post Print}

\section{Tweet}

N.B.: When citing this work, cite the original article.

Original Publication:

Daniel V Verschueren, Magnus P Jonsson and Cees Dekker, Temperature dependence of DNA translocations through solid-state nanopores, 2015, Nanotechnology, (26), 1-8.

http://dx.doi.org/10.1088/0957-4484/26/23/234004

Copyright: IOP Publishing: Hybrid Open Access

http://www.iop.org/

Postprint available at: Linköping University Electronic Press

http://urn.kb.se/resolve?urn=urn:nbn:se:liu:diva-121229 


\title{
Temperature dependence of DNA translocations through solid-state nanopores
}

\author{
Daniel V. Verschueren, Magnus P. Jonsson ${ }^{*}$ and Cees Dekke1 \\ Department of Bionanoscience, Kavli Institute of Nanoscience, \\ Delft University of Technology, Lorentzweg 1, 2628 CJ Delft, The Netherlands.
}

(Dated: June 23, 2015)

\begin{abstract}
In order to gain a better physical understanding of DNA translocations through solidstate nanopores, we study the temperature dependence of $\lambda$-DNA translocations through $10 \mathrm{~nm}$-in-diameter silicon-nitride nanopores, both experimentally and theoretically. The measured ionic conductance $G$, the DNA-induced ionic-conductance blockades $\Delta G$ and the event frequency $\Gamma$ all increase with increasing temperature while the DNA translocation time $\tau$ decreases. $G$ and $\Delta G$ are accurately described when bulk and surface conductances of the nanopore are considered and access resistance is incorporated appropriately. Viscous drag on the untranslocated part of the DNA coil is found to dominate the temperature dependence of the translocation times and the event rate is well described by a balance between diffusion and electrophoretic motion. The good fit between modeled and measured properties of DNA translocations through solid-state nanopores in this first comprehensive temperature study, suggest that our model captures the relevant physics of the process.
\end{abstract}

keywords: solids-state nanopores, temperature dependence, DNA translocations, nanopore conduction model.

\section{INTRODUCTION}

Solid-state nanopores are an emerging class of label-free, single-molecule biosensors, which are receiving great attention owing to their versatility and conceptual simplicity [1, 2]. At the heart of the nanopore sensor is a nanometer-sized hole, drilled in a thin silicon nitride membrane. Immersed between two containers filled with electrolyte, an ionic current can flow through this pore upon application of a bias voltage across the two containers. Charged biomolecules, such as DNA, are driven through the nanopore by the local electric field and partially obstruct the pore during passage. As a result, the current through the nanopore is reduced during this translocation, which

\footnotetext{
* Current address: Organic Electronics, Department of Science and Technology, Campus Norrköping, Linköping
} University, SE-60174 Norrköping, Sweden.

\footnotetext{
† Corresponding author: c.dekker@tudelft.nl
} 
provides the sensing signal for the single molecule. Nanopore devices have already found their way to a multitude of applications such as investigating DNA polymer physics [3, 4, anti-body binding kinetics [5], protein characterization [6 9], DNA depurination [10], and probing proteins bound on DNA [11-13].

Solid-state nanopore sensing systems have been integrated with laser optics in the past to affect DNA motion through the nanopore [14-16]. More recently, they have been combined with plasmonic optical antennas [17 21] to provide an alternative sensing method that complements the ionic current read-out. However, optical power concentration can lead to substantial local heating [22], most notably in plasmonic antennas [17, 23 26]. For such electro-optical nanopore systems, it is essential to understand how a temperature change affects the translocation process and nanopore conductance. A thorough understanding of the temperature dependence of the nanopore conductance will make it feasible to use the pore as a temperature probe with nanometer spatial resolution, for example, to quantify plasmonic heating from single nanostructures [25].

More generally, to extract biomolecular properties from a nanopore sensing experiment, it is vital to understand the fundamental physics that govern the translocation dynamics of the analyte. Previous investigations to elucidate these fundamentals have focused on voltage dependence [27, 28, DNA-length dependence [29], electrolyte ionic-strength dependence [30, 31] or a combination of those parameters [32], but the temperature dependence has remained largely uninvestigated. Some reports on DNA translocations through solid-state nanopores at different temperatures have been published [17, 32 34], including an extensive temperature analysis of DNA translocations through $\alpha$-HL pores [35]. Surprisingly however, a comprehensive study on the temperature dependence of DNA translocations through solid-state nanopores has not been published to date.

Four translocation characteristics can be extracted for a translocation experiment, as indicated in Figure 1A: the pore conductance $G$, the conductance drop produced by a translocating biomolecule $\Delta G$, the translocation time $\tau$, and the event rate $\Gamma$. Here we report the temperature dependence of these characteristics experimentally and we describe a model to explain our observations. 


\section{MATERIALS AND METHODS}

We fabricate nanopore devices as explained in [36]. Prior to measurements the chips were cleaned in an $\mathrm{O}_{2}$ plasma $(50 \mathrm{~W})$ for 1 minute. All experiments were performed on 8 to $16 \mathrm{~nm}$ diameter pores in $1 \mathrm{M} \mathrm{KCl}, 10 \mathrm{mM}$ Tris and $2 \mathrm{mM}$ EDTA buffer. DNA translocations were done with $\lambda$-DNA (Promega) at $\Delta V=100 \mathrm{mV}$ bias. To heat or cool the flow cell, a Peltier heater/cooler was used, as shown in Figure1B, with LABVIEW-controlled PID feedback. The temperature was monitored using two Pt100 thermistors, one directly placed on the flow cell and the other on the inner Faraday cage. For each newly set temperature, the flow cell was left to equilibrate for $20 \mathrm{~min}$ before acquisition. Acquisitions at different temperatures were carried out in random order and acquisition at room temperature $(296 \mathrm{~K})$ was always conducted at multiple occasions during an experiment, to prevent the convolution of changes due to temperature and residual temporal drifts in the system. Acquisition was done using an Axopatch 200B (Molecular Devices Ltd.) controlled by a custom-made LABVIEW program, and analysis of the data was done using a MATLAB-software package recently developed in our lab [37]. Pore diameters were obtained from TEM images (see Supporting Information (SI)) analyzed using ImageJ. All fitting is done by minimizing a $\chi^{2}$ goodness-of-fit parameter using a simple weighted linear regression, where $\chi^{2}=\frac{1}{N-2} \sum_{i=1}^{N}\left(\frac{y_{i}-\overline{y_{i}}}{s_{i}}\right)^{2}$. Here $N$ is the number of data points, $N-2$ the degrees of freedom in the fit (single fit parameter), $y_{i}$ is the experimental data point, $\bar{y}_{i}$ the corresponding predicted value by the fitted model and $s_{i}$ is the respective error.

\section{RESULTS}

\section{Open pore conduction}

Understanding the origins of the current through a nanopore is crucial for any nanopore experiment, as this current underlies the signal of the sensor. Figure 2 shows the experimental conductance (black dots) of a nanopore at different temperatures. The conductance of the pore is obtained from the slope of the linear $I-V$ curve taken between $+200 \mathrm{mV}$ and $-200 \mathrm{mV}$. There is a strong linear increase in the conductance of the pore with increasing temperature, showing a high sensitivity $\frac{\mathrm{d} G}{\mathrm{~d} T}=0.21 \mathrm{nS} / \mathrm{K}$ of the pore conductance to temperature, which represents a doubling of the pore current over a range of only $35 \mathrm{~K}$. 
Several efforts have already been made to elucidate the contributions to the ionic pore current analytically [16, 30, 38]. None of these models have been specifically tested through the temperature dependence of the current. Here, we describe our model. A rigorous derivation of the ionic conduction of the nanopore and its temperature dependence is provided in the SI.

Starting from the Poisson equation and the Nernst-Planck particle flux through a cylinder of diameter $d$ and length $l$ with a uniform surface charge density $\sigma$, one obtains (see SI) an expression for the pore conductance

$$
G_{c y l}=\left[\kappa_{b u l k} \frac{\pi d^{2}}{4 l}+\mu_{K}|\sigma| \frac{\pi d}{l}+\frac{\pi \sigma^{2} r_{D} d}{\eta l}\right]
$$

Here $\eta$ is the viscosity, $d$ is the pore diameter, $l$ is the pore length, $\kappa_{b u l k}$ is the bulk buffer conductivity of the electrolyte in the pore $\left(1 \mathrm{M} \mathrm{KCl}\right.$ in our case), $\mu_{K}$ and $\mu_{C l}$ are the mobilities for the two major charge carriers $\mathrm{K}^{+}$and $\mathrm{Cl}^{-}$respectively, and $r_{D}$ is the Debye length, which is the typical length scale over which the ion density significantly changes from the concentration in bulk.

Equation 1 shows the 3 main contributions to the nanopore conductance, as schematically indicated by the inset in Figure 2. By far the major contributor for these wide nanopores $(d \gtrsim 10 \mathrm{~nm})$ is the first term, the bulk conductance. Ions within the electrolyte are mobile, giving rise to this bulk conductance. The second term is the double-layer (DL) conductance. The electrolyte will act to screen surface charges of the silicon nitride by forming an excess layer of counterions at the pore surface [39]. Since the silicon-nitride surface charge density is negative, the (mobile) screening layer will be composed of potassium ions, which will move in the applied field, thus contributing a double-layer conductance. Finally, this net charge flow will set the background medium within the pore into motion. The flow that this creates, drags the charge of the double-layer, resulting in the electroosmotic flow (EOF) contribution to the pore conductance [16].

To model the conductance of a real nanopore, two more effects need to be accounted for: nanopores are in general not perfect cylinders and there is an access resistance that extends outside of the pore. Analogously to our previous work [40], we assume a total access resistance of $G_{\text {access }}{ }^{-1}=\left(\kappa_{\text {bulk }} d\right)^{-1}$ in series with a cylindrical pore resistance, and we use an effective pore length $l_{\text {eff }}$ to correct for the non-cylindrical shape of the nanopore. By evaluating the potential 
drop over the cylindrical pore, one finds the conductance of the nanopore to be

$$
\begin{aligned}
G & =\left[\kappa_{\text {bulk }}(T) \frac{\pi d^{2}}{4}+\mu_{K}(T)|\sigma(T)| \pi d+\frac{\pi \sigma^{2}(T) r_{D}(T) d}{\eta(T)}\right] \frac{1}{l_{\text {eff }}(1+\xi(T))}, \\
\xi(T) & =\frac{G_{\text {cyl }}}{G_{\text {access }}} .
\end{aligned}
$$

Here $\xi(T)$ is a parameter that indicates the ratio of the total conductance of the cylinder $G_{c y l}$ to the conductance of the access regions of the cylinder $G_{\text {access }}$. For a nanopore with $d=10 \mathrm{~nm}$ in $1 \mathrm{M} \mathrm{KCl}, \xi(T) \sim 1.1$ and only weakly dependent on temperature.

Figure 2 shows a fit of equation 2 to the experimental data. The different temperature-dependent parameters $\kappa_{\text {bulk }}(T), \mu_{k}(T), \sigma(T), r_{D}(T)$, and $\eta(T)$ are given in the SI. The effective length of the pore $l_{\text {eff }}$ is fitted, yielding a value of $7.0 \mathrm{~nm}$, which is very close to the value reported by [40] and in good agreement with the approximation by [41]. The model matches the experimental results well, considering that there is only a single fit parameter. Also shown are the three different components (bulk, double-layer and electroosmotic flow) that contribute to the nanopore conductance. It is clear that the bulk conductance constitutes the majority of the total pore conductance and that the EOF contribution is negligible.

\section{Blockade levels}

Biomolecules passing through the nanopore obstruct the pore partially and this volume exclusion produces a temporary reduction in the measured conductance. The magnitude of the conductance drops are the blockade levels $\Delta G$. A DNA molecule passing through a large nanopore will produce discrete blockade levels, depending on whether the molecule translocates in a linear fashion (only one double strand of DNA inside the pore) or in a folded fashion (two or more double strands of DNA in the pore simultaneously).

The blockade levels measured at various temperatures are fitted from a conductance histogram at each temperature and displayed in $3 \mathrm{~A}$, where the analysis was limited to only the first two blockade levels, one (red triangles) or two (red squares) double strands of DNA in the pore simultaneously. The blockade levels show a linear increase with temperature, doubling in size over about a $40 \mathrm{~K}$ range. Interestingly, this increase follows an almost identical trend as the open nanopore conductance, indicated by an almost constant $\Delta G / G$ in Figure $3 \mathrm{~B}$, although a small decrease in $\Delta G / G$ with temperature can be discerned. 
The conductance blockade originates from the DNA molecule partly obstructing the nanopore and the access region. This reduces the conductance of the bulk of the nanopore by volume exclusion. However, the access resistance is determined by the convergence of electric-field lines near the nanopore [42] and the modification of this resistance due the presence of the DNA molecule in this region is less obvious. We can approximate this situation by assuming that a similar volume exclusion term applies to the access regions, as done by Carlsen et al. [27]. A double strand of DNA will reduce the conductance of each access region by $\kappa_{b u l k} \frac{\pi d_{D N A}^{2}}{2 d}$, where $d_{D N A}$ is the diameter of a DNA strand and the approximate length of each access region is the radius of the pore $d / 2$. This then gives a total conductance of the access regions in the presence of a double strand of DNA as $G_{a c c \text { with } D N A}=\kappa_{\text {bulk }}\left(d-\frac{\pi d_{D N A}{ }^{2}}{4 d}\right)$.

Furthermore, a double-stranded DNA molecule is charged and this results in extra DL and EOF contribution due to ions flowing along the surface of the DNA molecule inside the nanopore, analogous to section III 1. Contrary to [27, we do not include a DNA surface conductance term in the access regions. Measurements done at low salt concentrations show that molecules in access region produce conductance blockades [28], whereas conductance enhancements are expected if the surface conductance of the DNA is included in this region. The conductance of the pore with a double strand of DNA inserted into the pore then becomes

$$
\begin{aligned}
G_{\text {with } D N A}= & {\left[\kappa_{\text {bulk }} \frac{\pi\left(d^{2}-d_{D N A}^{2}\right)}{4}+\left(|\sigma| d+\left|\sigma_{D N A}\right| d_{D N A}\right) \pi \mu_{K}\right.} \\
& \left.+\left(\sigma^{2} d+\sigma_{D N A}^{2} d_{D N A}\right) \frac{\pi r_{D}}{\eta}\right] \frac{1}{l_{e f f}\left(1+\xi_{\text {with } D N A}(T)\right)} .
\end{aligned}
$$

Here we have adopted a value of $0.034 \mathrm{Cm}^{-2}$ as the effective surface charge density of the DNA $\sigma_{D N A}$, which is a factor 4 smaller than the bare DNA surface charge density to account for counterion condensation 43 .

Note that $\xi_{\text {with } D N A}(T)$ for the nanopore containing DNA is about $1.5 \%$ larger than $\xi(T)$ for the open pore case, because the presence of the DNA strand affects the pore conductance and the access conductance differently. The conductance blockade that a single DNA duplex residing in the nanopore produces, is then given by $\Delta G=G_{\text {open pore }}-G_{\text {with } D N A}$. In the case two duplexes of DNA are present, the conductance blockade is almost exactly twice the blockade level for one double-strand of DNA.

Figure 3 A shows the predicted conductance blockade levels $\Delta G$ from equations 2 and 4 together with the experimental data, where we adopted a value of $7.0 \mathrm{~nm}$ for $l_{e f f}$, as extracted from the 
open pore conductance. The model agrees well with the experimental values, given the simple approximation to the change in access resistance and the absense of fit parameters.

The relative conductance blockade $\frac{\Delta G}{G}$, i.e., the conductance blockade level divided by the open pore conductance, provides yet another measure to test the model. The results for the two levels are shown in Figure $3 \mathrm{~B}$. The model predicts an almost constant value that approximates the experimental trend well, given that this involves no free fit parameters. The slight increase in the relative conductance blockades for lower temperatures is not well reproduced by the theoretical prediction, the small decrease of $\frac{\Delta G}{G}$ with increasing temperatures is in qualitative agreement with our previous observation [17].

\section{Translocation times}

Translocation times of analytes through nanopores has been a major topic of interest and it is known that decreasing the temperature increases the translocation time of the molecule [33]. Figure $3 \mathrm{C}$ displays the translocation time $\tau$ of $48.5 \mathrm{kbp} \lambda$-DNA molecules that pass through the nanopore in a linear fashion at different temperatures. There is a steep increase in translocation time for temperatures lower than room temperature and a slighter decrease in translocation times towards higher temperatures.

What sets the time it takes to translocate the molecule through a pore? Three main force contributions are at work during translocation [44]: (1) the electric field directly pulls on the charge on the DNA inside, (2) a viscous drag acts on the DNA inside the nanopore, and (3) a viscous drag exerts a force on the polymer blob outside the nanopore. The electric driving force acting on the DNA can be assumed to stay constant over the temperature range probed. The two retarding viscous forces couple to the molecule via the viscosity, which is strongly temperature dependent.

Inside the pore, the electroosmotic flow $v_{E O F}$ determines the magnitude of the drag force, because the flow speed is typically an order of magnitude larger than the translocation velocity $v_{t}$ of the DNA itself [45 47]. We assume a simple relation for the viscous drag $f_{d}=\gamma \eta v$, where $v$ is the velocity of the object with respect to the viscous medium and $\gamma$ is a drag coefficient depending

on the size of the object the drag force acts on. By using $v_{E O F}=\frac{r_{D} \sigma E_{z}}{\eta}$ [46] as the fluid velocity at the center of the pore, where $E_{z}$ is a constant electric field in the nanopore, the drag force that 
acts on the DNA inside the pore scales as

$$
f_{d} \propto \Delta V \sigma \frac{r_{D}}{l_{e f f}+\frac{\pi d}{4}}
$$

Here $\Delta V$ is the electric bias across the nanopore and the fraction $\frac{1}{l_{e f f}+\frac{\pi d}{4}}$ stems from the inclusion of access resistance in the analysis and from the non-cylindrical shape of the pore. Note that, importantly, this drag force contribution is independent of the viscosity [15, 45]. Since the remaining parameters in equation 5 all have a negligible dependence on temperature (see SI), this drag force is essentially independent of temperature. Hence the only force contribution that varies with temperature is the force on the polymer outside the nanopore.

Outside the pore, the conformation of the blob of the untranslocated DNA is important as it will determine the retention force. The exact shape of this untranslocated part during the translocation is debated and difficult to assess, even though models describing this have become increasingly more sophisticated [48]. We adopt a simple scaling model, as developed by Storm et al. [49], to fit to our results. Since in practice the Zimm relaxation time of the DNA coil is much slower than the translocation time of the molecule, the coil does not have time to relax during the translocation process. Hence polymer conformation at the moment of DNA capture into the pore, determines the drag that dictates the translocation time. The size of this DNA molecule is roughly the hydrodynamic radius of the whole coil, $R_{g}$, and it will take $\tau$ for this blob to travel over a distance equal to its own size $R_{g}$ to reach the pore. Hence $f_{d} \propto \eta \frac{R_{g}^{2}}{\tau}$ and since this drag balances the constant electrophoretic drive force, the translocation time is

$$
\tau \propto R_{g}^{2} \eta
$$

Both $R_{g}$ and $\eta$ are temperature dependent. $R_{g}$ depends on temperature because the persistence length of the DNA $a$ changes with temperature [50]. For a general coil, $R_{g} \propto a^{1-\nu}$ with $\nu$ the Flory exponent $(\approx 0.6$ in our case).

Figure $3 \mathrm{C}$ shows excellent agreement of the model to the experimental data (solid line, goodness-of-fit (see section II $\chi^{2}=2.3$ ), if we account for the temperature dependence of both $\eta$ and $R_{g}$. When the temperature dependence of the hydrodynamic radius is neglected, the fit is slightly worse (dashed line, $\chi^{2}=2.9$ ). It must be noted that the changes in persistence length are only moderate over the temperature range probed (ranging from $273 \mathrm{~K}$ to $313 \mathrm{~K}$ ) and the effect it has on the translocation time is weak. 


\section{Event rate}

One of the advantages of nanopores as single-molecule sensors is their ability to attract analytes, which increases the detection rate signifcantly compared sensors that rely on diffusion based transport. Figure $3 \mathrm{D}$ shows the event rate $\Gamma$, the rate at which molecules hit the pore and subsequently translocate through it. The event rate is observed to increase with increasing temperatures.

The rate at which DNA molecules pass through a nanopore is, for our experimental conditions, dictated by a balance between electrophoresis and diffusion [31]. The molecules diffusing within a region close to the pore will be attracted to the nanopore by an electrophoretic force that stems from the electric field emanating from the pore. Once the molecules enter this region, where the electrophoretic energy scale starts dominating over the diffusive energy scale, the molecules will be captured by the nanopore and will translocate through it. The size of this region determines the capture radius of the pore, which significantly exceeds the physical size of the pore, as shown by Grosberg and Rabin [31, 51]. If we include electrical access resistance and neglect surface charge effects such as electroosmotic flows, we arrive at an event rate

$$
\Gamma=c \Delta V \mu_{D N A} \frac{\pi d^{2}}{l_{e f f}+\frac{\pi d}{4}},
$$

where $c$ is the concentration of DNA in the cis chamber and $\mu_{D N A}$ is the electrophoretic mobility of the DNA.

The temperature dependence of the event rate is contained in the electrophoretic mobility $\mu_{D N A}$ of DNA. Since the DNA coil is freely draining, i.e. the solvent molecules move through the DNA coil upon application of an electric force, the mobility is determined by a local balance between the electric pull and the viscous drag on the DNA. For a small Debye length $r_{D}$, appropriate for our high-salt conditions, this dependence can be approximated by [52]

$$
\mu_{D N A} \propto \frac{r_{D}}{\eta} .
$$

Figure $3 \mathrm{D}$ shows the event rate changing with temperature and indicates a decent fit of the model. Because of fragmentation of long DNA polymers during handling, the concentration of $\lambda$-DNA molecules in the cis chamber is not accurately known and equation 7 is fitted, with $c$ as a fit parameter. The normalized $\chi^{2}$ value of the fit is 60 , which is a large value, but it must be noted that the deviations in the trend are most likely set by small variations in pore diameter, which are not accounted for in the fitting error values. Using $d=8.1 \mathrm{~nm}, l_{e f f}=7.0 \mathrm{~nm}$ as appropriate for 
this data set, and $\mu_{D N A} \approx 2.2 \cdot 10^{10} \mathrm{~nm}^{2} /(\mathrm{V} \cdot \mathrm{s})$ [51], we find an effective concentration of $\lambda$-DNA of $0.4 \mathrm{ng} / \mu \mathrm{L}$. This is lower than the $5 \mathrm{ng} / \mu \mathrm{L}$ of $\lambda$-DNA used in the initial sample and we attribute the discrepancy to fragmentation and the uncertainty in our numerical estimate for $\mu_{D N A}$.

\section{DISCUSSION}

Changing the temperature of the system affects many parameters. Because molecular interactions that give rise to collective properties, such as viscosity and permittivity, are in general temperature dependent, each of these features should be carefully inspected to explain the temperature dependence of DNA translocations. The SI contains a list of all parameters that exhibit or are expected to exhibit an appreciable temperature dependence. Even though the list is substantial, the main trends are captured by only considering the temperature dependence of the viscosity and the buffer conductivity.

In high-ionic-strength solutions, charges are in general not only screened by counterions, but also effectively reduced, by counterion condensation [43]. The degree of condensation can depend on temperature. Remarkably, however, the models reproduce the experimental trends very well while keeping the effective charge of the DNA constant. The results indicate that the counterion condensation on the DNA does not vary very much within the temperature range probed (273 $318 \mathrm{~K}$ ) in these high-ionic-strength solutions.

While all trends were measured in multiple experiments on multiple pores, the data displayed in each separate figure are taken from a single experiment, to prevent slight changes in experimental conditions. The translocation times and event rates of all experiments can be found in the SI.

\section{CONCLUSION}

We have presented here the first extensive study on the temperature dependence on DNA translocation through solid-state nanopores. We analyzed the characteristic features of these translocations, comprising the open pore conductance $G$, conductance blockade $\Delta G$, translocation times $\tau$, and event rate $\Gamma$. In order to explain the observed experimental trends in these features, we have extended existing theory by incorporating the access resistance in established models. The temperature dependence of $G$ and $\Delta G$ are well described when the conductance contributions from the bulk and the surface charges of the nanopore walls and the DNA inside the 
pore are taken into account. The open pore conduction of the nanopore is very sensitive to local changes in temperature and can be used to determine heating from nanostructures. Additionally, the trends in event rate $\Gamma$ could be accurately described by a balance between diffusion and electrophoretic motion [51]. Finally, for the translocation time $\tau$ we obtained excellent agreement with a model developed by Storm et al. [49, where the untranslocated coil dictates the translocation velocity of the DNA molecule.

\section{ACKNOWLEDGEMENTS}

We would like to acknowledge Xander Janssen for building the temperature-regulated nanopore setup. Alexei Aksimentiev and his group as well as Tim Albrecht are acknowledged for the valuable discussions. We thank Meng-Yue Wu for drilling nanopores and Calin Plesa for his help in translocation data analysis. This work was supported by the National Human Genome Research Institute of the National Institute of Health under Award Number 1R01HG007406-01, by a WennerGren Fellowship from the Wenner-Gren Foundations, by an ERC Advanced grant NanoforBio (no. 247072) and by the Netherlands Organisation for Scientific Research (NWO/OCW), as part of the Frontiers of Nanoscience program.

[1] Cees Dekker. Solid-state nanopores. Nat Nano, 2(4):209-215, 2007. 1748-3387 10.1038/nnano.2007.27 10.1038/nnano.2007.27.

[2] Meni Wanunu. Nanopores: A journey towards dna sequencing. Physics of Life Reviews, 9(2):125-158, 2012. 1571-0645.

[3] Calin Plesa, Ludo Cornelissen, Maarten W. Tuijtel, and Cees Dekker. Non-equilibrium folding of individual dna molecules recaptured up to 1000 times in a solid state nanopore. Nanotechnology, 24(47):475101, 2013.

[4] Mirna Mihovilovic, Nicholas Hagerty, and Derek Stein. Statistics of dna capture by a solid-state nanopore. Physical Review Letters, 110(2):028102, 2013. 1079-7114.

[5] Ruoshan Wei, Volker Gatterdam, Ralph Wieneke, Robert Tampe, and Ulrich Rant. Stochastic sensing of proteins with receptor-modified solid-state nanopores. Nat Nano, 7(4):257-263, 2012. 1748-3387 10.1038/nnano.2012.24 10.1038/nnano.2012.24.

[6] Calin Plesa, Stefan W. Kowalczyk, Ruben Zinsmeester, Alexander Y. Grosberg, Yitzhak Rabin, and Cees Dekker. Fast translocation of proteins through solid state nanopores. Nano Letters, 13(2):658-663, 
2013.

[7] Matthias Firnkes, Daniel Pedone, Jelena Knezevic, Markus Dblinger, and Ulrich Rant. Electrically facilitated translocations of proteins through silicon nitride nanopores: conjoint and competitive action of diffusion, electrophoresis, and electroosmosis. Nano Letters, 10:2162-7, 2010.

[8] Gautam V. Soni and Cees Dekker. Detection of nucleosomal substructures using solid-state nanopores. Nano Letters, 12(6):3180-3186, 2012.

[9] Erik C Yusko, Jay M Johnson, Sheereen Majd, Panchika Prangkio, Ryan C Rollings, Jiali Li, Jerry Yang, and Michael Mayer. Controlling protein translocation through nanopores with bio-inspired fluid walls. Nat Nano, 6:253-60, 2011.

[10] Michael M. Marshall, Jan A. Ruzicka, Ethan W. Taylor, and Adam R. Hall. Detecting dna depurination with solid-state nanopores. PLoS ONE, 9(7):e101632, 2014.

[11] Stefan W. Kowalczyk, Adam R. Hall, and Cees Dekker. Detection of local protein structures along dna using solid-state nanopores. Nano Letters, 10(1):324-328, 2009. doi: 10.1021/nl903631m 1530-6984 doi: $10.1021 / \mathrm{nl} 903631 \mathrm{~m}$.

[12] C. Raillon, P. Cousin, F. Traversi, E. Garcia-Cordero, N. Hernandez, and A. Radenovic. Nanopore detection of single molecule rnapdna transcription complex. Nano Letters, 12(3):1157-1164, 2012.

[13] Deanpen Japrung, Azadeh Bahrami, Achim Nadzeyka, Lloyd Peto, Sven Bauerdick, Joshua B. Edel, and Tim Albrecht. Ssb binding to single-stranded dna probed using solid-state nanopore sensors. The Journal of Physical Chemistry B, 118(40):11605-11612, 2014.

[14] Ulrich F. Keyser, Bernard N. Koeleman, Stijn van Dorp, Diego Krapf, Ralph M. M. Smeets, Serge G. Lemay, Nynke H. Dekker, and Cees Dekker. Direct force measurements on dna in a solid-state nanopore. Nat Phys, 2(7):473-477, 2006. 10.1038/nphys344.

[15] Stijn van Dorp, Ulrich F. Keyser, Nynke H. Dekker, Cees Dekker, and Serge G. Lemay. Origin of the electrophoretic force on dna in solid-state nanopores. Nat Phys, 5(5):347-351, 2009. 10.1038/nphys1230.

[16] Nicolas Di Fiori, Allison Squires, Daniel Bar, Tal Gilboa, Theodore D. Moustakas, and Amit Meller. Optoelectronic control of surface charge and translocation dynamics in solid-state nanopores. Nat Nano, 8(12):946-951, 2013.

[17] Francesca Nicoli, Daniel Verschueren, Misha Klein, Cees Dekker, and Magnus P. Jonsson. Dna translocations through solid-state plasmonic nanopores. Nano Letters, 2014.

[18] Michael P. Cecchini, Aeneas Wiener, Vladimir A. Turek, Hyangh Chon, Sangyeop Lee, Aleksandar P. Ivanov, David W. McComb, Jaebum Choo, Tim Albrecht, Stefan A. Maier, and Joshua B. Edel. Rapid ultrasensitive single particle surface-enhanced raman spectroscopy using metallic nanopores. Nano Letters, 13(10):4602-4609, 2013.

[19] Yi Li, Chang Chen, Sarp Kerman, Pieter Neutens, Liesbet Lagae, Guido Groeseneken, Tim Stakenborg, and Pol Van Dorpe. Harnessing plasmon-induced ionic noise in metallic nanopores. Nano Letters, 
13(4):1724-1729, 2013.

[20] SungWoo Nam, Inhee Choi, Chi-cheng Fu, Kwanpyo Kim, SoonGweon Hong, Yeonho Choi, Alex Zettl, and Luke P. Lee. Graphene nanopore with a self-integrated optical antenna. Nano Letters, 14(10):5584$5589,2014$.

[21] Joseph Larkin, Mathieu Foquet, Stephen W. Turner, Jonas Korlach, and Meni Wanunu. Reversible positioning of single molecules inside zero-mode waveguides. Nano Letters, 14(10):6023-6029, 2014.

[22] Ulrich F. Keyser, Diego Krapf, Bernard N. Koeleman, Ralph M. M. Smeets, Nynke H. Dekker, and Cees Dekker. Nanopore tomography of a laser focus. Nano Letters, 5(11):2253-2256, 2005.

[23] Nadine Harris, Michael J. Ford, and Michael B. Cortie. Optimization of plasmonic heating by gold nanospheres and nanoshells. The Journal of Physical Chemistry B, 110(22):10701-10707, 2006.

[24] Zachary J. Coppens, Wei Li, D. Greg Walker, and Jason G. Valentine. Probing and controlling photothermal heat generation in plasmonic nanostructures. Nano Letters, 13(3):1023-1028, 2013.

[25] Magnus P. Jonsson and Cees Dekker. Plasmonic nanopore for electrical profiling of optical intensity landscapes. Nano Letters, 13(3):1029-1033, 2013.

[26] Joseph B. Herzog, Mark W. Knight, and Douglas Natelson. Thermoplasmonics: Quantifying plasmonic heating in single nanowires. Nano Letters, 14(2):499-503, 2014.

[27] Autumn T. Carlsen, Osama K. Zahid, Jan Ruzicka, Ethan W. Taylor, and Adam R. Hall. Interpreting the conductance blockades of dna translocations through solid-state nanopores. ACS Nano, 8(5):47544760, 2014.

[28] Stefan W. Kowalczyk and Cees Dekker. Measurement of the docking time of a dna molecule onto a solid-state nanopore. Nano Letters, 12(8):4159-4163, 2012.

[29] A. J. Storm, J. H. Chen, H. W. Zandbergen, and C. Dekker. Translocation of double-strand dna through a silicon oxide nanopore. Physical Review E: Statistical, Nonlinear, and Soft Matter Physics, 71(5):051903, 2005. PRE.

[30] Ralph M. M. Smeets, Ulrich F. Keyser, Diego Krapf, Meng-Yue Wu, Nynke H. Dekker, and Cees Dekker. Salt dependence of ion transport and dna translocation through solid-state nanopores. Nano Letters, 6(1):89-95, 2006. doi: 10.1021/nl052107w 1530-6984 doi: 10.1021/nl052107w.

[31] Meni Wanunu, Will Morrison, Yitzhak Rabin, Alexander Y. Grosberg, and Amit Meller. Electrostatic focusing of unlabelled dna into nanoscale pores using a salt gradient. Nat Nano, 5(2):160-165, 2010. 10.1038/nnano.2009.379.

[32] Meni Wanunu, Jason Sutin, Ben McNally, Andrew Chow, and Amit Meller. Dna translocation governed by interactions with solid-state nanopores. Biophysical Journal, 95(10):4716-4725, 2008.

[33] Daniel Fologea, James Uplinger, Brian Thomas, David S McNabb, and Jiali Li. Slowing dna translocation in a solid-state nanopore. Nano Letters, 5:1734-7, 2005. 
[34] Yin Zhang, GenSheng Wu, Jian Ma, ZhiShan Yuan, Wei Si, Lei Liu, JingJie Sha, and YunFei Chen. Temperature effect on translocation speed and capture rate of nanopore-based dna detection. Science China Technological Sciences, pages 1-7, 2014.

[35] Amit Meller, Lucas Nivon, Eric Brandin, Jene Golovchenko, and Daniel Branton. Rapid nanopore discrimination between single polynucleotide molecules. Proceedings of the National Academy of Sciences, 97(3):1079-1084, 2000.

[36] Xander J. A. Janssen, Magnus P. Jonsson, Calin Plesa, Gautam V. Soni, Cees Dekker, and Nynke H. Dekker. Rapid manufacturing of low-noise membranes for nanopore sensors by trans -chip illumination lithography. Nanotechnology, 23(47):475302, 2012. 0957-4484.

[37] Calin Plesa and Cees Dekker. Data analysis methods for solid-state nanopores; submitted. 2014.

[38] T. Albrecht, T. Gibb, and P. Nuttall. Chapter 1 - Ion Transport in Nanopores, pages 1-30. William Andrew Publishing, Oxford, 2013.

[39] Reto B. Schoch, Jongyoon Han, and Philippe Renaud. Transport phenomena in nanofluidics. Reviews of Modern Physics, 80(3):839-883, 2008. RMP.

[40] Stefan W. Kowalczyk, Alexander Y. Grosberg, Yitzhak Rabin, and Cees Dekker. Modeling the conductance and dna blockade of solid-state nanopores. Nanotechnology, 22(31):315101, 2011. 0957-4484.

[41] Meni Wanunu, Tali Dadosh, Vishva Ray, Jingmin Jin, Larry McReynolds, and Marija Drndic. Rapid electronic detection of probe-specific micrornas using thin nanopore sensors. Nat Nano, 5(11):807-814, 2010. 10.1038/nnano.2010.202.

[42] James E Hall. Access resistance of a small circular pore. The Journal of general physiology, 66(4):531$532,1975$.

[43] Gerald S. Manning. The molecular theory of polyelectrolyte solutions with applications to the electrostatic properties of polynucleotides. Quarterly Reviews of Biophysics, 11(02):179-246, 1978.

[44] Lei Chen and A. T. Conlisk. Forces affecting double-stranded dna translocation through synthetic nanopores. Biomedical Microdevices, 13(2):403-414, 2011.

[45] Sandip Ghosal. Electrokinetic-flow-induced viscous drag on a tethered dna inside a nanopore. Physical Review E, 76(6):061916, 2007. PRE.

[46] C. T. A. Wong and M. Muthukumar. Polymer capture by electro-osmotic flow of oppositely charged nanopores. The Journal of Chemical Physics, 126(16):-, 2007.

[47] Binquan Luan and Aleksei Aksimentiev. Electro-osmotic screening of the dna charge in a nanopore. Physical Review E, 78(2):021912, 2008. PRE.

[48] Payam Rowghanian and Alexander Y. Grosberg. Force-driven polymer translocation through a nanopore: An old problem revisited. The Journal of Physical Chemistry B, 115(48):14127-14135, 2011. 
[49] Arnold J. Storm, Cornelis Storm, Jianghua Chen, Henny Zandbergen, Jean-Francois Joanny, and Cees Dekker. Fast dna translocation through a solid-state nanopore. Nano Letters, 5(7):1193-1197, 2005. doi: 10.1021/nl048030d 1530-6984 doi: 10.1021/nl048030d.

[50] Stephanie Geggier, Alexander Kotlyar, and Alexander Vologodskii. Temperature dependence of dna persistence length. Nucleic Acids Research, 39(4):1419-1426, 2011.

[51] Alexander Y. Grosberg and Yitzhak Rabin. Dna capture into a nanopore: Interplay of diffusion and electrohydrodynamics. The Journal of Chemical Physics, 133(16):-, 2010.

[52] Payam Rowghanian and Alexander Y. Grosberg. Electrophoresis of a dna coil near a nanopore. Physical Review E, 87(4):042723, 2013. PRE. 
A

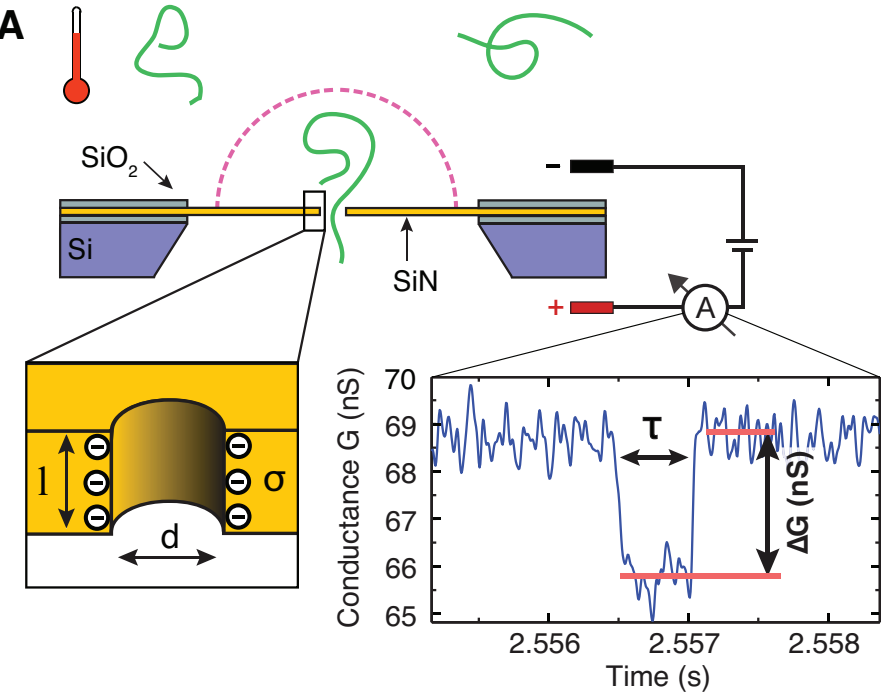

B

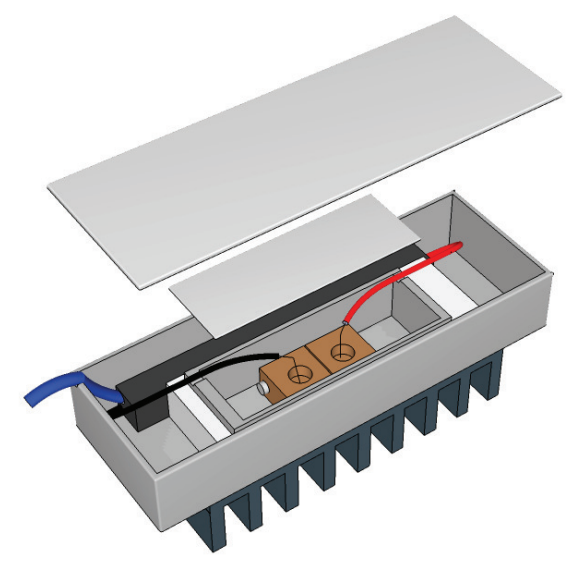

FIG. 1: A: schematics of a nanopore experiment. A nanometer-sized hole of diameter $d$ is drilled into a thin silicon-nitride membrane of thickness $l$. During an experiment the conductance $G$ of the nanopore is monitored and modulated by $\Delta G$ upon passage of a DNA molecule. $G, \Delta G$, the translocation time $\tau$ and the event rate $\Gamma$ (the capture radius is depicted as the pink dashed hemisphere) are investigated here as a function of temperature. B Experimental setup consisting of two Faraday cages and a peltier heater/cooler. The PEEK flow cell is located in the inner Faraday cage, the head-stage of the amplifier in the outer. 


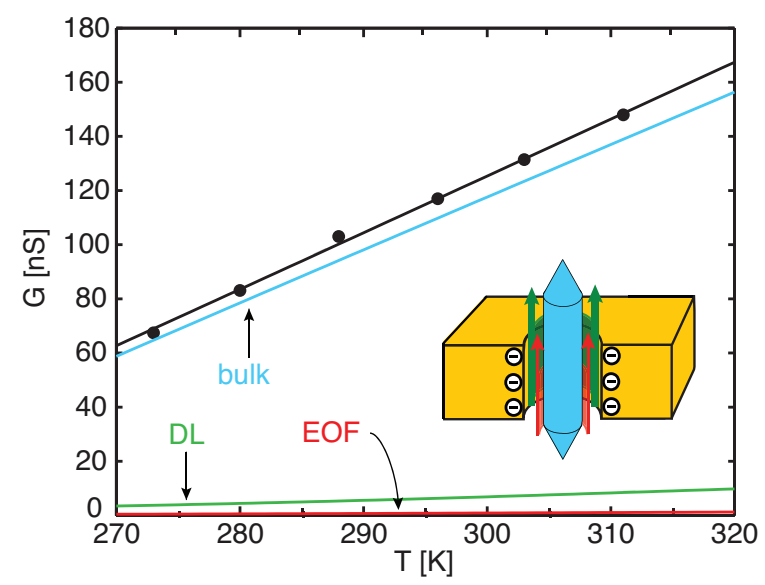

FIG. 2: Temperature dependence of the conductance $G$ of a $d=16 \mathrm{~nm}$ nanopore in $1 \mathrm{M} \mathrm{KCl}$. The black dots are experimental data points and the solid black line is a fit from equation 2 . The inset shows the different contributions to the current. The bulk conductance is displayed in blue, and the surface conductances from the double-layer (DL) and electroosmotic flow (EOF) are displayed in green and red respectively. 
A

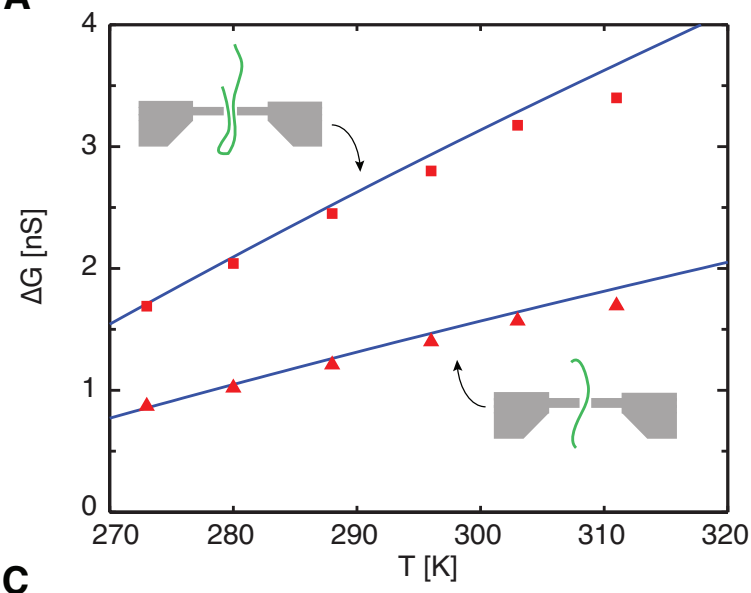

C

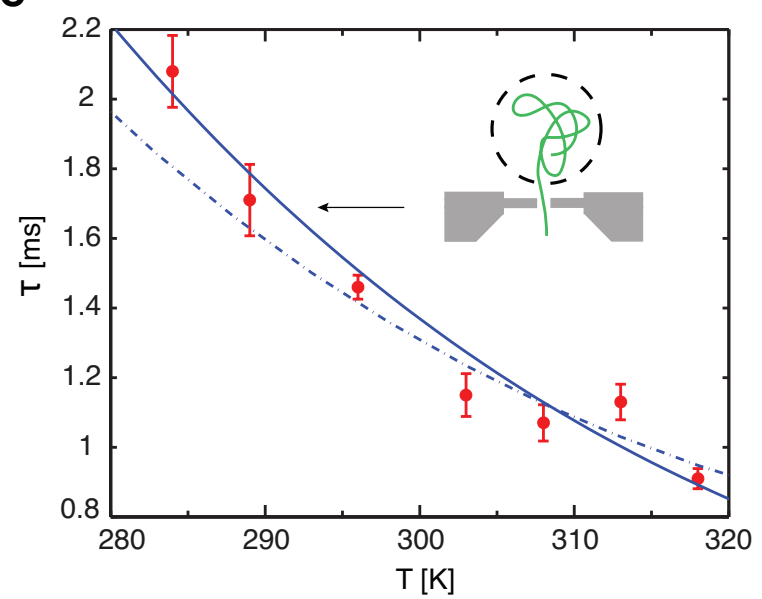

B

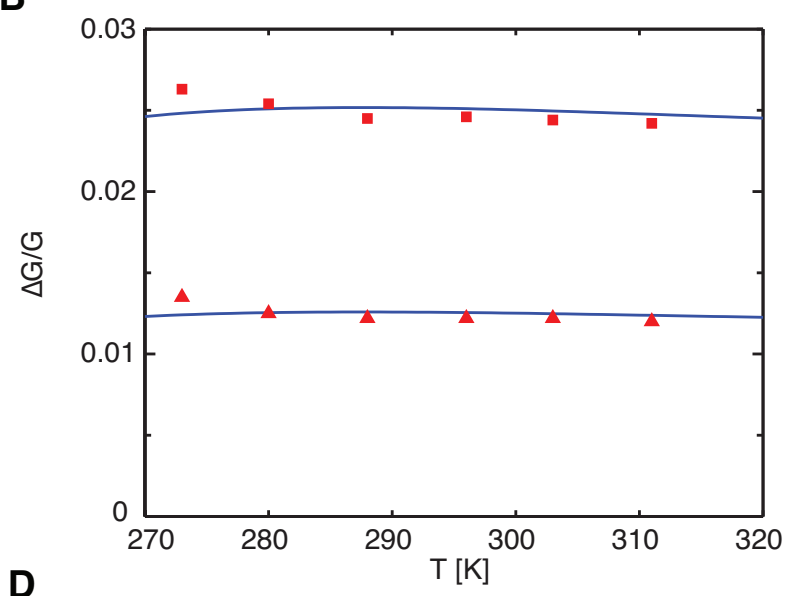

D

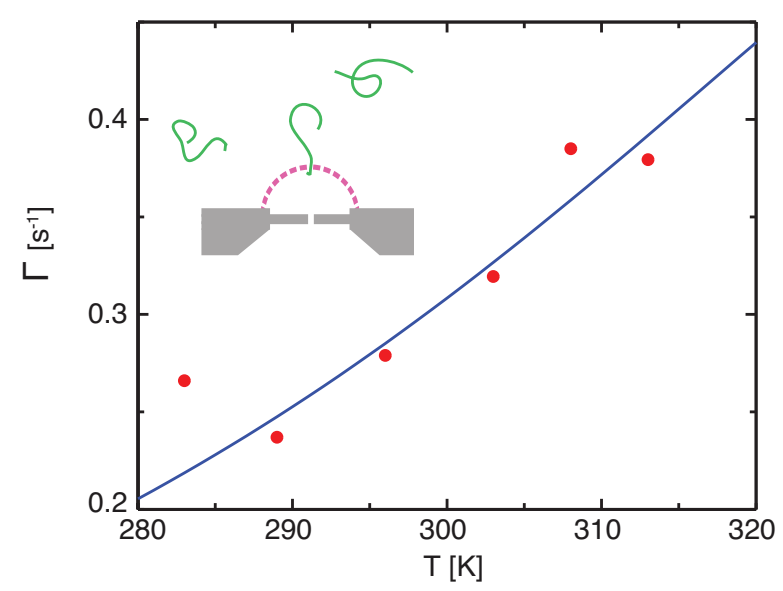

FIG. 3: Characteristics of DNA translocations as a function of temperature. A: Conductance blockades $\Delta G$ for linear (triangles) and folded (squares) translocations in a $d=16 \mathrm{~nm}$ pore. The blue lines are the predictions from equations 2 and 4 for a single blockade and a double blockade level, using $l_{e f f}=7 \mathrm{~nm}$ as obtain from the open pore conductance. B: Relative conductance blockade levels $\frac{\Delta G}{G}$ from the same pore as in A. Blue lines are obtained from the fits of $G$ and $\Delta G$. C Translocation times $\tau$ for $\lambda$-DNA in a $d=8.1 \mathrm{~nm}$ pore. The solid line is a fit of equation $6\left(\chi^{2}=2.3\right)$, the dashed line is a fit to $t_{d} \propto \eta\left(\chi^{2}=2.9\right)$. D Event rate for $\lambda$-DNA in a $d=8.1 \mathrm{~nm}$ pore. Solid line is a fit to equation $7\left(\chi^{2}=60\right)$. The symbols in this figure are larger than the errorbars. Experimental data from figures $\mathbf{C}$ and $\mathbf{D}$ are reprinted with permission from [17]. Copyright 2014 American Chemical Society. 


\section{Supporting Information}

Temperature dependence of DNA translocations through solid-state nanopores

Daniel V. Verschueren, Magnus P. Jonsson ${ }^{*}$ and Cees Dekker

Department of Bionanoscience, Kavli Institute of Nanoscience, Delft University of Technology, Lorentzweg 1, 2628 CJ Delft, The Netherlands.

1 Nanopore conduction model

2 Temperature dependence of parameters in conduction model

3 TEM images of nanopores

4 Translocation times and event rates from different experiments

* Current address: Organic Electronics, Department of Science and Technology, Campus Norrköping, Linköping University, SE-60174 Norrköping, Sweden. 


\section{NANOPORE CONDUCTION MODEL}

The electrical current through a nanopore is determined by a steady state flux of ions through the small orifice. This ion flux is governed by three fundamental equations. The Nernst-Planck flux (equation S.1) describes the particle flux $\mathbf{J}_{i}(\mathbf{r})$ of each species $i$ directly. The ion density $n_{i}(\mathbf{r})$ is not only determined by this equation, but also by the Poisson equation (equation $\mathrm{S} .2$ ), as the ions are charged. The background medium can in general support an incompressible Stokes flow $\mathbf{u}(\mathbf{r})$ (equation S.3), as the Reynolds number is small for flow at these dimensions.

$$
\begin{aligned}
\mathbf{J}_{i}(\mathbf{r}) & =D_{i} \nabla n_{i}(\mathbf{r})-\mathbf{u}(\mathbf{r}) n_{i}(\mathbf{r})+\frac{D_{i} z_{i} e}{k_{B} T} n_{i}(\mathbf{r}) \nabla \phi(\mathbf{r}) \\
\nabla^{2} \phi(\mathbf{r}) & =-\frac{\rho(\mathbf{r})}{\epsilon} \\
\eta \nabla^{2} \mathbf{u}(\mathbf{r}) & =-\rho(\mathbf{r}) \nabla \phi(\mathbf{r})
\end{aligned}
$$

Here $D_{i}$ is the diffusion constant of species $i, z_{i}$ is it's valence, $\epsilon$ is the electrical permittivity, $\eta$ is the viscosity of the background medium, $\rho(\mathbf{r})=\sum_{i} z_{i} e n_{i}(\mathbf{r})$ is the volume charge density, and $\phi(\mathbf{r})$ the electric potential.

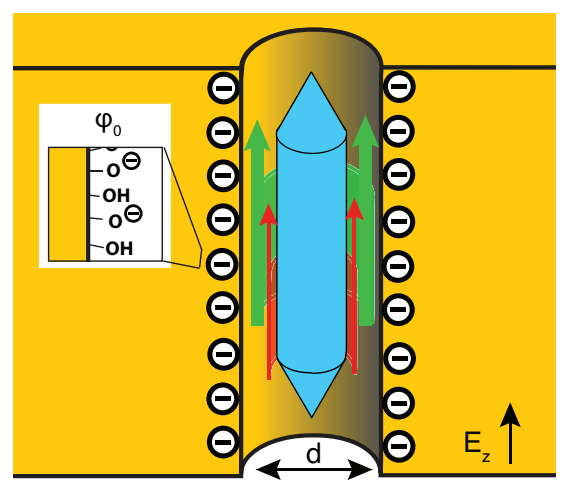

Fig. S.1: Schematic of cylinder of diameter d, filled with electrolyte embedded in silicon nitride. The cylinder has a uniform $\zeta$-potential $\phi_{0}$ due to the deprotonated silanol groups at the surface and an axial electric field $E_{z}$ is applied. The bulk conductance (blue arrow) and the surface conductances, double-layer (DL, green arrow) and electroosmotic flow conductances (EOF, red arrow) are indicated in the figure.

First we consider a simplified system, containing solely a perfect cylinder filled with electrolyte. The cylinder has a diameter $d$, the walls are at a uniform $\zeta$-potential of $\phi_{0}$, and a constant axial electric field $\frac{\partial \phi(\mathbf{r})}{\partial z}=E_{z}$ is applied. A schematic of this is shown in Fig. S.1. The cylinder contains a conductive electrolyte with two major charge carriers: potassium and chloride, each with mobility $\mu_{K}$ and $\mu_{C l}$ respectively. 
The symmetry in the radial direction dictates the flow and the net ion-flux to be zero in this direction. This simplifies the PNP equations to the Poisson-Boltzmann (PB) equation ,.

$$
\nabla^{2} \phi(r)=-\frac{1}{\epsilon} \sum_{i} z_{i} n_{0} \exp \left(-\frac{z_{i} e \phi(r)}{k_{B} T}\right) .
$$

For a single-valence binary electrolyte, the PB equation sets a typical length scale $r_{D}=\sqrt{\frac{\epsilon k_{B} T}{2 n_{0} e^{2}}}$, the Debye length. This is the distance over which the ion density significantly changes from the bulk density $n_{0}$. In case the Debye length is small compared to the scale of the system $\left(r_{D} \ll d\right)$, we can linearize the exponential term in equation S.4. The PB equation then simplifies to the DebyeHückel limit, for which equation S.4 can be solved analytically, invoking cylindrical symmetry [1]:

$$
\phi(r)=\phi_{0} \frac{I_{0}\left(r / r_{D}\right)}{I_{0}\left(d / 2 r_{D}\right)} .
$$

Here, $I_{0}(x)$ is the zero-order modified Bessel-function of the first kind.

With this solution to the electric potential, equation S.3 can readily be solved. The symmetry restricts the net flow to be along the axis of the cylinder and only the $z$ component of the flow is non-zero. With a no-slip condition on the wall of the cylinder, $u_{z}(r)$ becomes [2]

$$
u_{z}(r)=-\frac{\epsilon \phi_{0} E_{z}}{\eta}\left[1-\frac{I_{0}\left(r / r_{D}\right)}{I_{0}\left(d / 2 r_{D}\right)}\right] .
$$

Using the Debye-Hückel approximation, the current density becomes:

$$
J_{z, e}=\sum_{i=K, C l} z_{i} e J_{z, i}=\left(\mu_{K}+\mu_{C l}\right) e n_{0} E_{z}-\mu_{K} \frac{\epsilon \phi(r)}{r_{D}^{2}} E_{z}-u_{z}(r) \frac{\epsilon \phi(r)}{r_{D}^{2}}
$$

where we used the Einstein relation $\mu_{i}=\frac{e D_{i}}{k_{B} T}$. Combining equations S.5, S.6, and S.7, we have a complete analytic expression for the current density inside the cylinder. Fig. S 2 shows the current density distribution due to the separate terms in this equation for a $d=10 \mathrm{~nm}$ cylinder with an electric field density $E_{z}=5 \cdot 10^{7} \mathrm{~V} / \mathrm{m}$ and a $\zeta$-potential of $\phi_{0}=-14 \mathrm{mV}$, which corresponds to the silicon-nitride $\zeta$-potential at room temperature (see Fig. S.5B)

We can assign a physical interpretation to each term in equation $S .7$ if we identify $\frac{\epsilon \phi(r)}{r_{D}^{2}}$ as an effective volume charge density. The first term represents the bulk current density. The second term is the double-layer (DL) current density as the volume charge density itself is mobile and affected by the electric field. The last term in this expression is the electroosmotic flow (EOF) current, which arises from a net charge flux due to the flow in the background medium $u_{z}(r)$. 


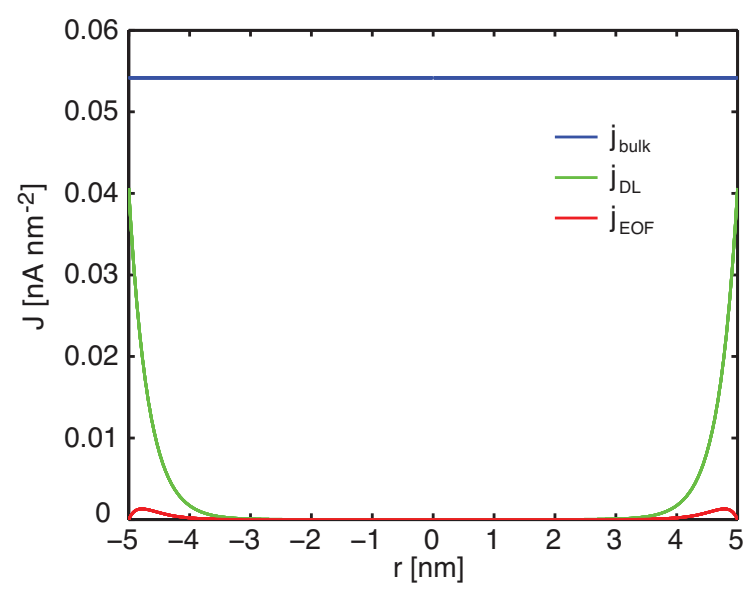

Fig. S.2: Current density distribution inside the cylinder. The bulk current density $j_{b u l k}$ is by far the dominant contribution over the surface currents, double-layer current density $j_{D L}$ and EOF current density $j_{E O F}$.

A note must be made to the double-layer contribution, which arises from the screening of the surface charge density on the walls of the cylinder. The Debye-Hückel theory predicts, incorrectly, that the reduction of coion density near the charged cylinder wall is equal and opposite to the enhancement of counterion density. This is not true close to the charged surface and is borne out by the full Poisson-Boltzmann theory [3]. A common approximation is to neglect the reduction of coions entirely and attribute the screening exclusively to the counterions, as is done here in equation S.7.

Integrating the expression for the current density over the cylinder yields the total current through it. For this we need $\int_{0}^{x} x^{\prime} I_{0}\left(x^{\prime}\right) \mathrm{d} x^{\prime}=x I_{1}(x)$, where $I_{1}(x)$ is roughly equal to $I_{0}(x)$ for large $x\left(d / 2 r_{D} \gg 1\right)$ and $\int_{0}^{x} x^{\prime} I_{0}^{2}\left(x^{\prime}\right) \mathrm{d} x^{\prime}=\frac{1}{2} x^{2}\left(I_{1}^{2}(x)-I_{0}^{2}(x)\right)$, which approximates to 0 for large $x$. The resulting current in terms of $\phi_{0}$ is then

$$
\begin{aligned}
I_{c y l}= & \int_{0}^{\frac{d}{2}} J_{z, e}(r) 2 \pi r \mathrm{~d} r= \\
& n_{0} e\left(\mu_{K}+\mu_{C l}\right) \frac{\pi d^{2}}{4} E_{z}+\mu_{K} \epsilon\left|\phi_{0}\right| \frac{\pi d}{r_{D}} E_{z}+\frac{\pi \epsilon^{2} \phi_{0}^{2} d}{\eta r_{D}} E_{z} .
\end{aligned}
$$

A more familiar expression can be obtained by rewriting $\phi_{0}$ to a surface charge density $\sigma$. The surface charge density is related to the $\zeta$-potential by the Grahame equation

$$
\sigma\left(\phi_{0}\right)=\frac{2 k_{B} T \epsilon}{r_{D} e} \sinh \left(\frac{e \phi_{0}}{2 k_{B} T}\right)
$$

In the case $e\left|\phi_{0}\right|$ is small with respect to $k_{B} T$, this equation reduces to $\sigma=\frac{\epsilon \phi_{0}}{r_{D}}$. We can then 
rewrite equation $\mathrm{S} .8$

$$
I_{c y l}=[\overbrace{n_{0} e\left(\mu_{K}+\mu_{C l}\right)}^{\kappa_{b u l k}} \frac{\pi d^{2}}{4}+\mu_{K}|\sigma| \pi d+\frac{\pi \sigma^{2} r_{D} d}{\eta}] E_{z} .
$$

When access resistance is neglected, $E_{z}$ is simply $\frac{\Delta V}{l}$ where $\Delta V$ is the bias voltage applied across the cylinder of length $l$. The conductance of the cylinder then becomes

$$
G_{c y l}=\left[\kappa_{b u l k} \frac{\pi d^{2}}{4 l}+\mu_{K}|\sigma| \frac{\pi d}{l}+\frac{\pi \sigma^{2} r_{D} d}{\eta l}\right] .
$$

The first term in equation S.11 is the bulk conductance $G_{b u l k}$ and the last two terms make up the surface conductance of the pore: the conductance due to the double-layer $G_{D L}$ and the conductance due to the flow in the medium $G_{E O F}$. This equation is in agreement with other expressions for the pore current, derived with more heuristic methods [4], where only [6] takes the small EOF contribution into account.

In the case of a real nanopore, the shape deviates from that of a perfect cylinder and access effects need to be taken care of. Kowalzcyk et al. [7] have shown that a nanopore is in general not a cylinder, but hourglass shaped and included access resistance in the conduction model. This implies that $E_{z}$ is not just $\frac{\Delta V}{l}$, but that only part of the voltage drop is over the cylindrical pore, which now has a modified thickness $l_{\text {eff }}$. Assuming a total access resistance for both regions of $G_{\text {access }}=d \kappa_{\text {bulk }}$, the voltage drop over the pore becomes:

$$
\Delta V_{\text {pore }}=\frac{G_{c y l}^{-1}}{{G_{c y l}}^{-1}+G_{\text {access }}^{-1}} \Delta V
$$

and subsequently the electric field strength in the pore becomes

$$
E_{z}=\frac{\Delta V}{l_{e f f}(1+\xi(T))}
$$

Here, $\xi(T)=\frac{G_{c y l}}{G_{a c c e s s}}$ is roughly equal to 1.1 in our conditions, and depends weakly on temperature. Hence, the conductance of the pore is:

$$
G_{\text {pore }}=\left[\kappa_{\text {bulk }} \frac{\pi d^{2}}{4}+\mu_{K}|\sigma| \pi d+\frac{\pi \sigma^{2} r_{D} d}{\eta}\right] \frac{1}{l_{e f f}(1+\xi(T))}
$$

\section{TEMPERATURE DEPENDENCE OF PARAMETERS IN CONDUCTION MODEL}

Many parameters in the model as described in section 1 are temperature dependent. This section lists all the temperature dependent parameters used in the model. 
1. Viscosity $\eta$, mobility $\mu_{K}$, and conductivity $\kappa_{\text {bulk }}$

A

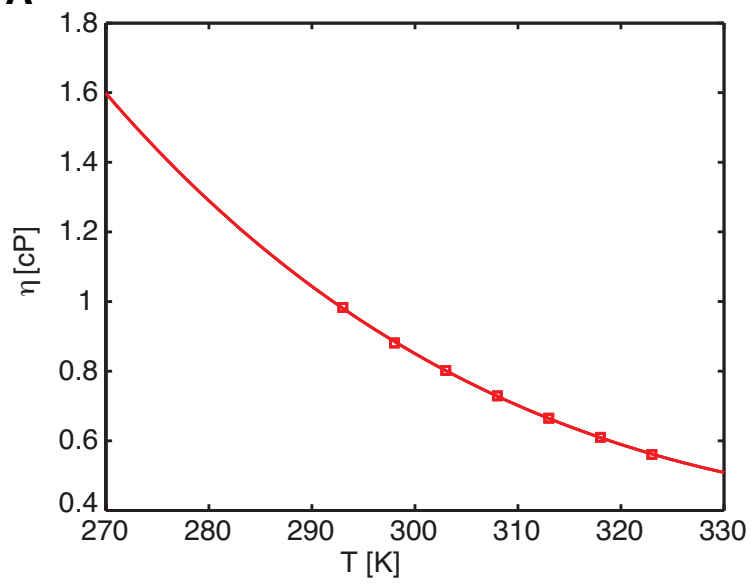

B

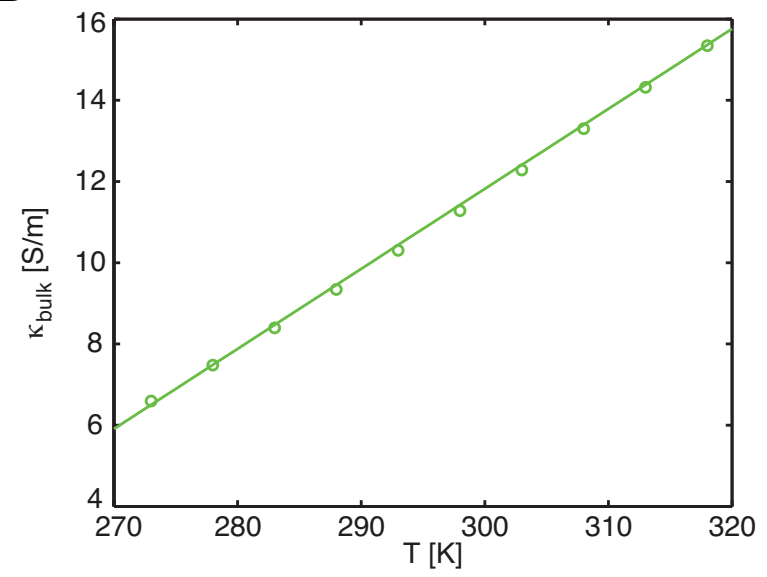

Fig. S.3: A Temperature dependence of viscosity of $1 \mathrm{M} \mathrm{KCl}$ and the respective fit. B Temperature dependence of the buffer conductivity of $1 \mathrm{M} \mathrm{KCl}$ and the respective fit.

The temperature dependence on the viscosity $\eta$ is fitted to data obtained from [8] with a second order polynomial in $T^{-1}$. The temperature dependence of the bulk conductivity of the electrolyte is obtained from [9] and fitted to a linear function of $T$ [10].

The temperature dependence of the mobility of the potassium ions, which make up the doublelayer conductance, is assumed to depend directly on the viscosity:

$$
\mu_{K}(T)=\mu_{K}(T=296 K) \frac{\eta(T=296 K)}{\eta(T)},
$$

where $\mu_{K}(T=296 K)=7.910^{-8} \mathrm{~m}^{2}(\mathrm{Vs})^{-1}$.

\section{Debye length $r_{D}$}

To calculate the temperature dependence of the Debye length $r_{D}=\sqrt{\frac{k_{B} T \epsilon}{2 n_{0} e^{2}}}$, we used a temperature-dependent electric permittivity [11]. The change of $r_{D}$ with temperature is actually minor, as shown in Fig. S4.

\section{Surface charge density $\sigma$}

The surface charge density in the pore is set by the silanol chemistry of the $\mathrm{SiN}[12$. The $\zeta$ potential is related to the surface charge density by the chemical equilibrium of the surface silanol 


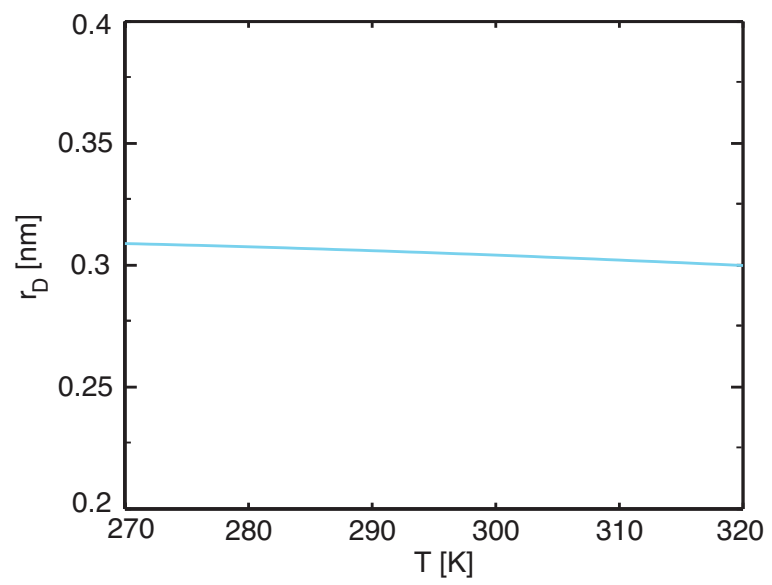

Fig. S.4: Temperature dependence of the Debye length $r_{D}$ in $1 \mathrm{M} \mathrm{KCl}$.
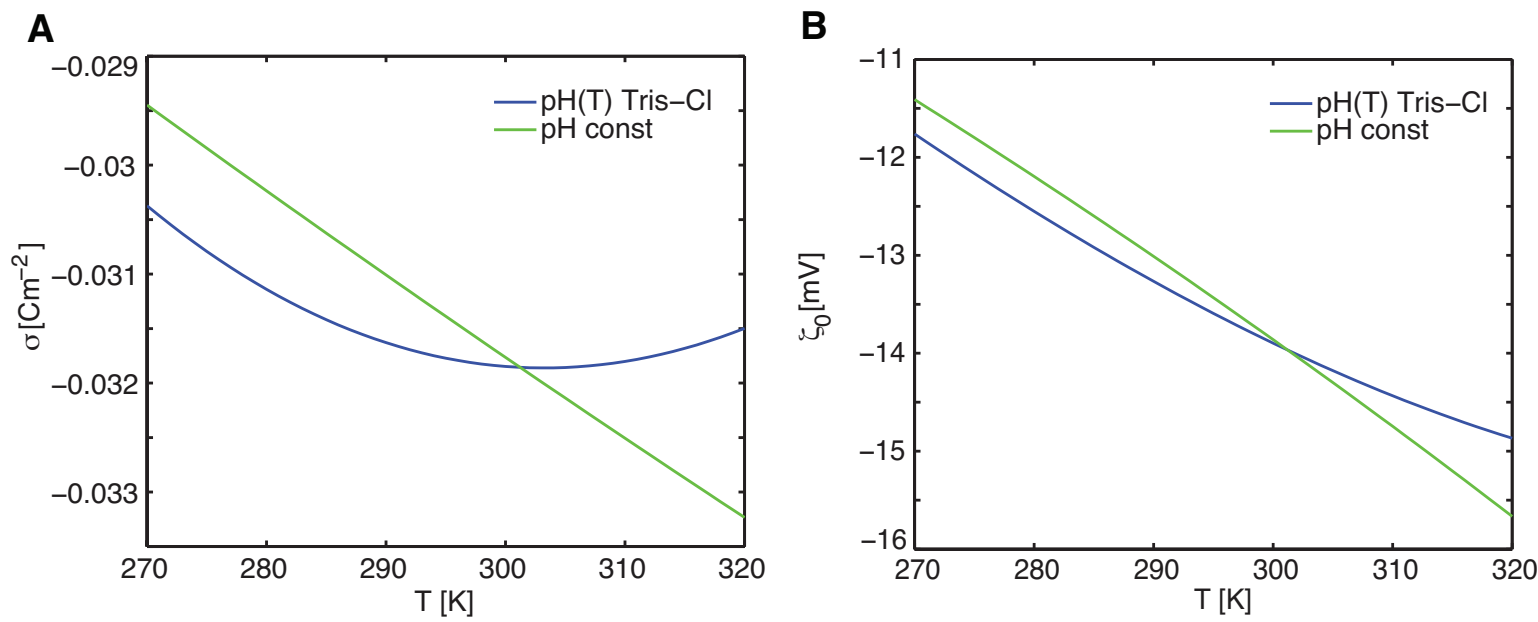

Fig. S.5: A Temperature dependence of the surface charge density $\sigma$ on the silicon nitride according to the coupled Grahame, Behrens-Grier equations, with a constant $\mathrm{pH}$ (green) and a temperature dependent pH, as for Tris- $\mathrm{Cl}$ (blue). B Temperature dependence of the $\zeta$-potential $\phi_{0}$. Again the effect of changing the $\mathrm{pH}$ (blue) or keeping the $\mathrm{pH}$ constant with temperature (green) is shown.

groups, as described by the Behrens-Grier equation [13]

$$
\phi_{0}(\sigma)=\frac{k_{B} T}{e} \ln \left(\frac{-\sigma}{e \gamma+\sigma}\right)-(\mathrm{pH}-\mathrm{pK}) \frac{k_{B} T}{e} \ln (10)-\frac{\sigma}{C},
$$

where $\gamma$ is the site density of the surface silanol group $\left(8 \mathrm{~nm}^{-2}\right)$, pK it's acid dissociation constant, and $C$ the stern capacitance of the surface $\left(0.3 \mathrm{Fm}^{-2}\right)$ [14]. Together with the Grahame equation (equation S.9), the surface charge density $\sigma$ can be solved self-consistently, using an iterative method like, for example, MATLAB's fsolve().

$\mathrm{pK}$ and $\mathrm{pH}$ values are in general temperature dependent. In this case, pK of the surface silanol 
groups is $\mathrm{pK}=-\frac{\Delta G^{*}}{2.3 N_{A} k_{B} T}$. Here $\Delta G^{*}=4.2 \mathrm{~kJ} / \mathrm{mol}$ [15] is the Gibbs free energy gain of the dissociation of a proton from a silanol surface group. In case of the $\mathrm{pH}$ of Tris-Cl, the temperature dependence is $\mathrm{pH}(T)=8.0-0.03 \cdot(T-296)[16]$.

Fig. S 5 shows the temperature dependence of both the $\zeta$-potential and surface charge density $\sigma$ of the pore's surface, where the $\mathrm{pH}$ is kept constant with temperature or allowed to vary as the $\mathrm{pH}$ of Tris-Cl. It is clear from this figure that the choice for Tris-Cl buffer causes very little change in the surface charge density.

\section{TEM IMAGES OF NANOPORES}

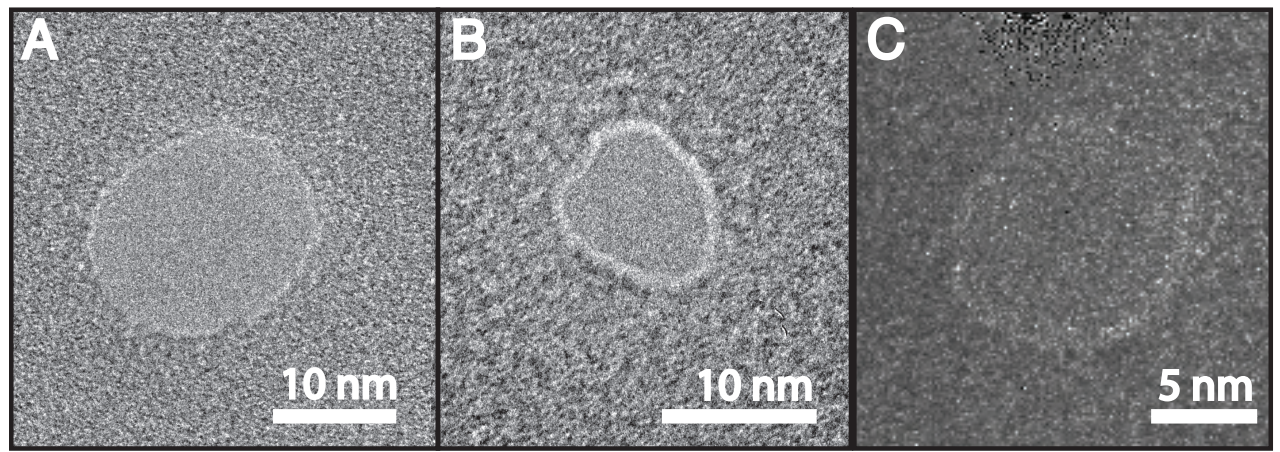

Fig. S.6: A pore TB4 $12-9(d=16.0 \mathrm{~nm})$ B pore TB1A 3-9 $(d=8.1 \mathrm{~nm})$ C pore TB1B $1-9(d=8.9 \mathrm{~nm})$

Figure 2 and figures 3A and $\mathrm{B}$ in the main text and Fig. S.7. A and $\mathrm{C}$ are data from pore TB4 12-9 (Fig. S6A), figures 3C and D in the main text are from pore TB1A 3-9 (Fig. S6B) and Fig. S.7B and D are from pore TB1B 1-9 (Fig. S.6C). 


\section{TRANSLOCATION TIMES AND EVENT RATES FROM DIFFERENT EXPERIMENTS}

A

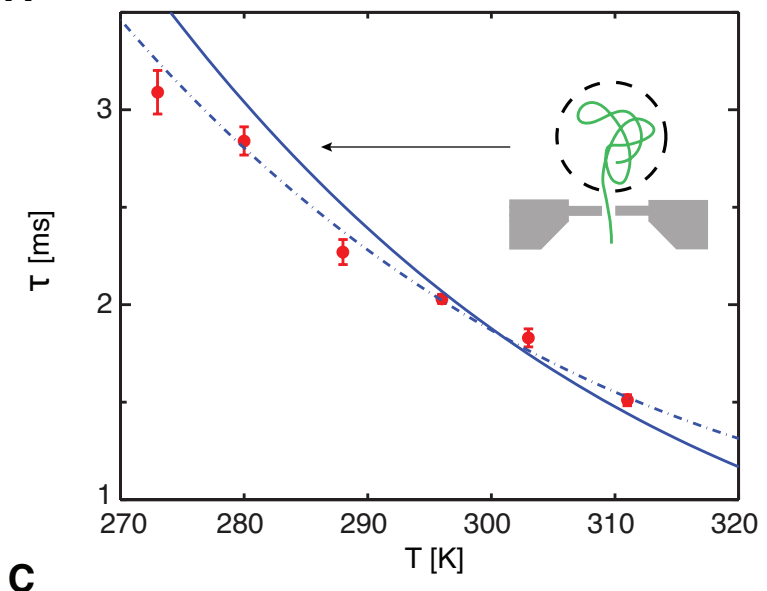

C

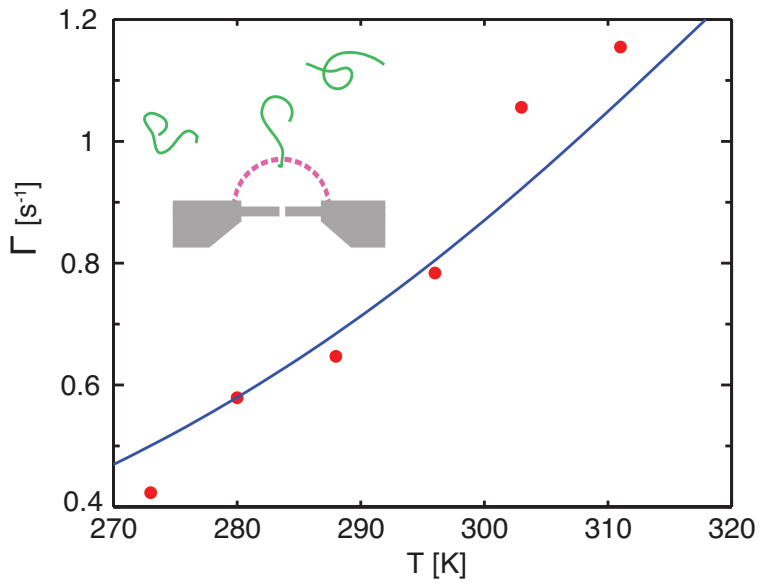

B
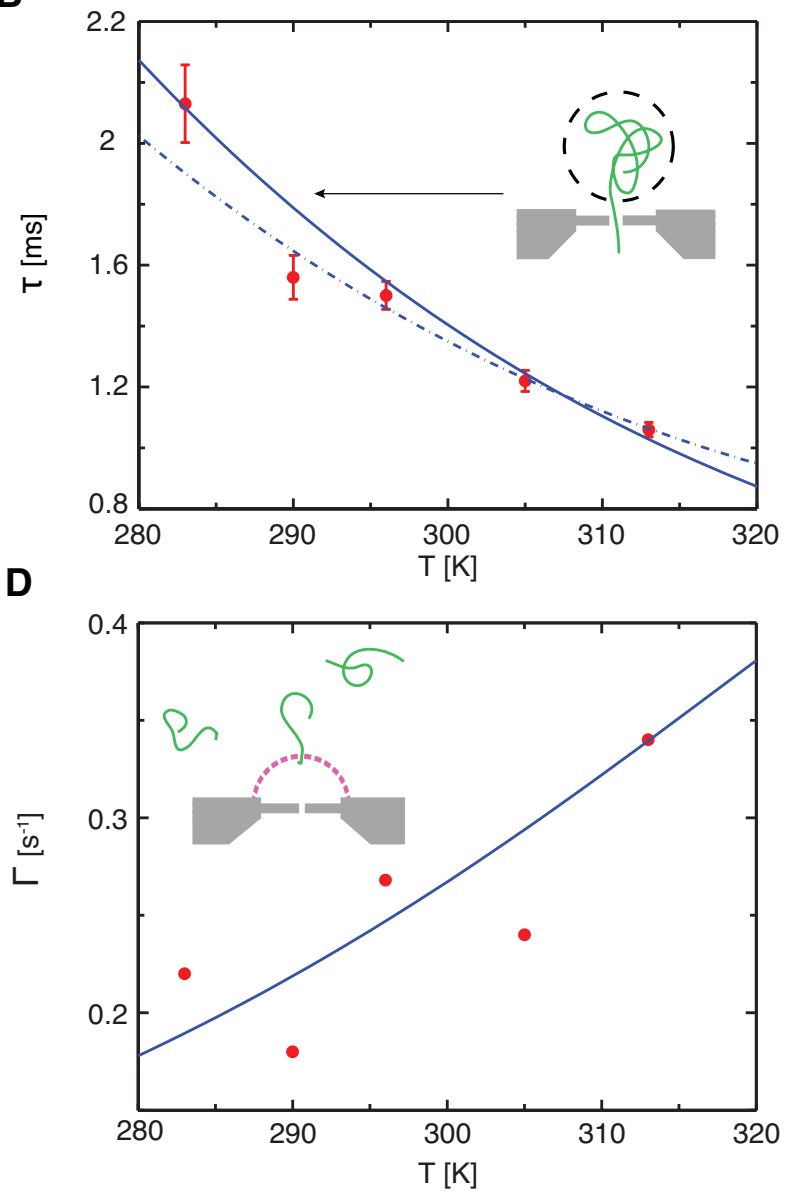

Fig. S.7: Event rate $\Gamma$ and translocation time $\tau$ of $\lambda$-DNA translocations as a function of temperature. A: Translocation times $\tau$ for TB4 12-9. $\chi^{2}=9.1$ (solid line) from fit of equation 6 in the main text, $\chi^{2}=1.7$ (dashed line) from fit of viscosity only. B: Translocation times $\tau$ for TB1B 1-9. $\chi^{2}=2.9$ (solid line), $\chi^{2}=1.7$ (dashed line). C Event rate $\Gamma$ for TB4 12-9. The solid line is a fit of equation 7 in the main text, $\chi^{2}=480$. The symbol size is large than the error bars. D Event rate $\Gamma$ for TB4 $12-9, \chi^{2}=640$. The symbol size is large than the error bars.

[1] F. Booth. The solution of some potential problems in the theory of electrolytes. The Journal of Chemical Physics, 19(7):821-826, 1951.

[2] C. T. A. Wong and M. Muthukumar. Polymer capture by electro-osmotic flow of oppositely charged 
nanopores. The Journal of Chemical Physics, 126(16):-, 2007.

[3] Reto B. Schoch, Jongyoon Han, and Philippe Renaud. Transport phenomena in nanofluidics. Reviews of Modern Physics, 80(3):839-883, 2008. RMP.

[4] Ralph M. M. Smeets, Ulrich F. Keyser, Diego Krapf, Meng-Yue Wu, Nynke H. Dekker, and Cees Dekker. Salt dependence of ion transport and dna translocation through solid-state nanopores. Nano Letters, 6(1):89-95, 2006. doi: 10.1021/nl052107w 1530-6984 doi: 10.1021/nl052107w.

[5] T. Albrecht, T. Gibb, and P. Nuttall. Chapter 1 - Ion Transport in Nanopores, pages 1-30. William Andrew Publishing, Oxford, 2013.

[6] Nicolas Di Fiori, Allison Squires, Daniel Bar, Tal Gilboa, Theodore D. Moustakas, and Amit Meller. Optoelectronic control of surface charge and translocation dynamics in solid-state nanopores. Nat Nano, 8(12):946-951, 2013.

[7] Stefan W. Kowalczyk, Alexander Y. Grosberg, Yitzhak Rabin, and Cees Dekker. Modeling the conductance and dna blockade of solid-state nanopores. Nanotechnology, 22(31):315101, 2011. 0957-4484.

[8] M. Afzal, M. Saleem, and M. Tariq Mahmood. Temperature and concentration dependence of viscosity of aqueous electrolytes from 20.degree.c to 50.degree.c chlorides of (sodium(1+), potassium(1+), magnesium $(2+)$, calcium $(2+)$, barium $(2+)$, strontium $(2+)$, cobalt $(2+), \operatorname{nickel}(2+), \operatorname{copper}(2+)$ and chromium(3+). Journal of Chemical E Engineering Data, 34(3):339-346, 1989.

[9] K. W. Pratt, W. F. Koch, Y. C. Wu, and P. A. Berezansky. Molality-based primary standards of electrolytic conductivity (iupac technical report), 2001.

[10] Magnus P. Jonsson and Cees Dekker. Plasmonic nanopore for electrical profiling of optical intensity landscapes. Nano Letters, 13(3):1029-1033, 2013.

[11] G. C. Malmberg and A. A. Maryott. Dielectric constant of water from 0c to 100c. Journal of Research of the National Bureau of Standards, 56(1), 1956.

[12] Nicole Jaffrezic-Renault, Amitabha De, Paul Clechet, and Abderrazak Maaref. Study of the silicon nitride/aqueous electrolyte interface on colloidal aqueous suspensions and on electrolyte/insulator/semiconductor structures. Colloids and Surfaces, 36(1):59-68, 1989.

[13] Sven H. Behrens and David G. Grier. The charge of glass and silica surfaces. The Journal of Chemical Physics, 115(14):6716-6721, 2001.

[14] Frank HJ van der Heyden, Derek Stein, and Cees Dekker. Streaming currents in a single nanofluidic channel. Physical review letters, 95(11):116104, 2005.

[15] A. Revil, P. A. Pezard, and P. W. J. Glover. Streaming potential in porous media: 1. theory of the zeta potential. Journal of Geophysical Research: Solid Earth, 104(B9):20021-20031, 1999.

[16] Inc. New England BioLabs. ph vs temperature for tris buffer, 2014. 